\title{
Simple setup for wavelength estimation by using the Airy disk with didactical purposes
}

\section{U. Rivera-Ortega}

U. Rivera-Ortega, "Simple setup for wavelength estimation by using the Airy disk with didactical purposes," Proc. SPIE 10741, Optics Education and Outreach V, 107410P (14 September 2018); doi: 10.1117/12.2306652

EDIE Event: SPIE Optical Engineering + Applications, 2018, San Diego, California, United States 


\title{
Simple setup for wavelength estimation by using the Airy disk with didactical purposes
}

\author{
U. Rivera-Ortega ${ }^{a}$
}

\author{
${ }^{a}$ Facultad de Ciencias de la Electrónica, Benemérita Universidad Autónoma del Estado de Puebla, \\ CU, Boulevard 18 Sur, Av San Claudio \& Jardines de San Manuel, Puebla, Pue. México
}

\begin{abstract}
In this manuscript a simple and easy to implement method that uses the Airy disk generated from a Fraunhofer diffraction pattern due to a circular aperture will be used to estimate the wavelength of the illuminating laser source. This estimation is based on the measurement of the Airy disk diameter, whose approximation is directly proportional to the wavelength of the light source and to the distance between the aperture and the image plane; and inversely proportional to the diameter of the aperture. Due to the characteristics and versatility of the present proposal, this is perfectly suitable to be applied in graduate or undergraduate physics laboratories or even in classrooms for educational and/or demonstrative purposes.
\end{abstract}

Keywords: Wavelength, Airy disk, didactic-resource, automation

\section{INTRODUCTION}

There are some suggested methods that use optical setups to measure the wavelength of a laser source, which in the visible spectrum goes from 400 to 700 nanometers $(\mathrm{nm})$ corresponding to a color range from violet through red [1-4]. Some of them are based on interferometers which count the number of fringes or the spacing between them [5-7], while some others use the distance between the diffraction orders generated by a diffractive element such as a diffraction grating [8] or a ruler [9]. In this manuscript, common electronics and low-cost optical elements such as a circular aperture and a generic laser diode source will be used to provide an easy implementation with the aim of estimating the wavelength of the source, with didactical purposes.

When a uniform beam of light passes through a circular aperture, a resulting Airy patter which is a diffraction pattern formed by a series of concentric bright rings rounding a central bright spot can be observed. The bright region in the center is known as the Airy disk [10-12], which contains approximately the $84 \%$ of the total incoming energy, as seen in Figure 1

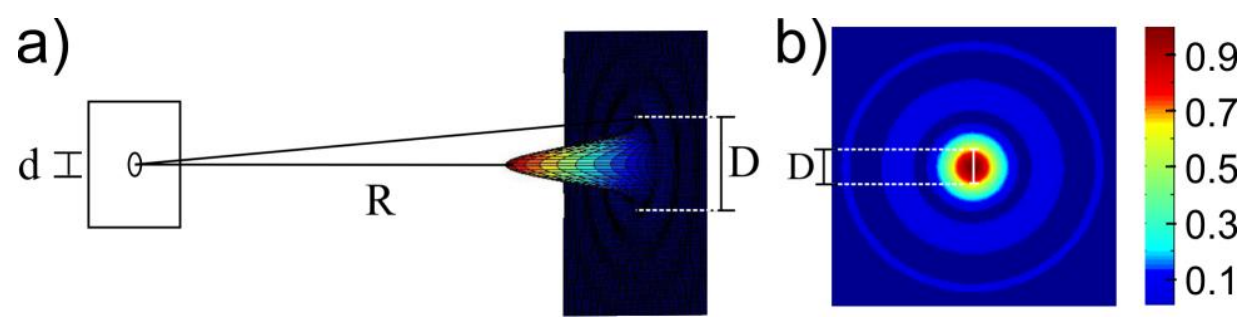

Figure 1. a) Diffraction patter generated by a circular aperture at a distance R, b) the Airy disk of diameter D.

The relationship between the diameter of the Airy disk and the wavelength of the laser source is given by

$$
D=\frac{2.44 \lambda R}{d}
$$


so that

$$
\lambda=\frac{d r}{1.22 R}
$$

where $\lambda$ is the wavelength of the source, $d$ is the diameter of the aperture and $R$ is the distance between the aperture and the observation plane.

In our experience, it is very common to demonstrate in graduate or undergraduate physics laboratories the relationship shown in Equation (1), where the student is commonly required to measure the diameter of the disk resulting from different distances $R$ and apertures $d$ and in some cases with different laser wavelengths [15]; but as far as we know it has never been used directly to give an estimation of the wavelength of the source.

In the present proposal a simple method that uses the diameter of an Airy disk belonging to the Fraunhofer far field diffraction pattern generated by a circular aperture will be applied to estimate the wavelength of a laser source, according to Equation (2).

\section{METHODOLOGY}

The experimental setup of the proposal is shown in Figure 2a), in which due to the relation between the size of the selected pinhole (a circular aperture of diameter $d=100 \mu \mathrm{m}$ ) and its distance to the visualization screen $R$, the resulting Airy pattern meets the characteristics corresponding to a Fraunhofer diffraction [16-17]. The light source in this setup is a collimated beam from a laser diode connected to a voltage source. To store the images, a Webcam Logitech-C920 has been used. Figure $2 b$ ) shows the ring mount where a piece of vellum paper has been used as a visualization screen. It will be shown that the size of the inner diameter of this mount was used to calibrate the system, so the size of the Airy disk in pixels must be transformed in real spatial units $(\mathrm{cm})$.

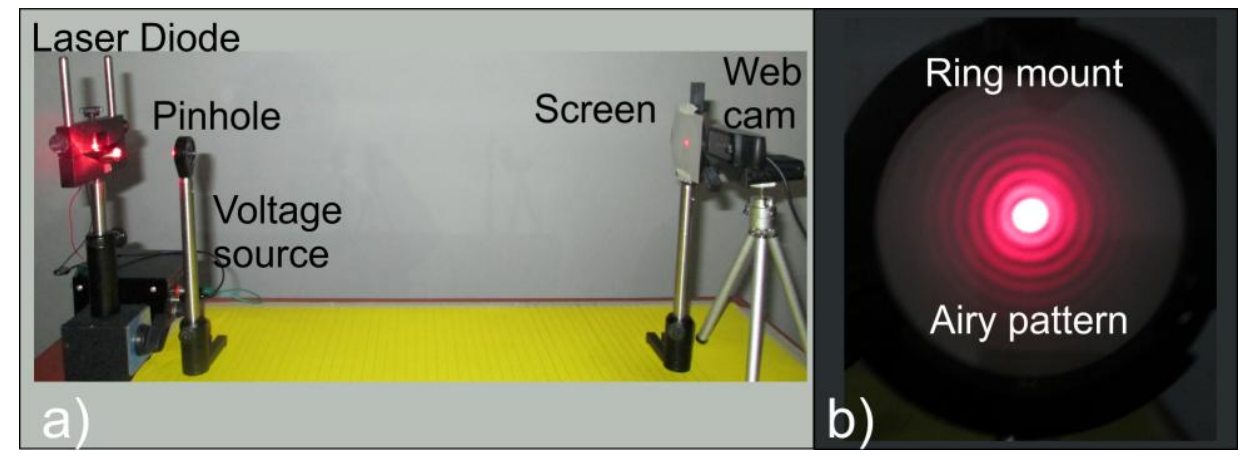

Figure 2. a) Experimental setup, b) experimental Airy pattern.

In order to compute the measurements in spatial units, an interactive MATLAB calibration procedure has been developed (nevertheless, all the following measurement procedure can be done manually directly from the observation plane). To accomplish this task, a known reference distance was selected. For this particular case the inner diameter of the ring mount $(5.8 \mathrm{~cm})$ was used in which the visualization screen has been attached; however any known distance (solid line in Figure 3a)) can be selected by clicking on its initial and final position. Once the real spatial unit and the distance have been typed by the user in the input box (Figure 3b), its equivalence in pixels and the ratio of the selected unit per pixel will be displayed (Figure 3c)). 


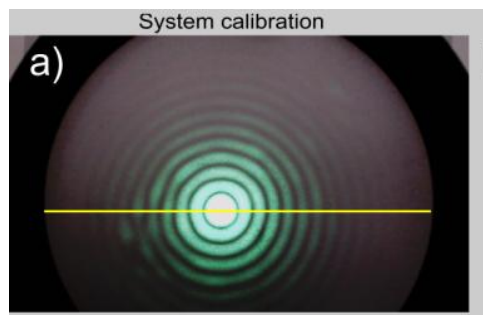

b)

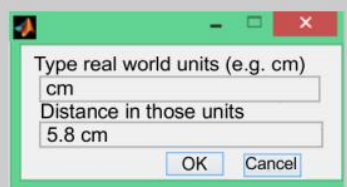

c)

\section{or} The selected distance is 2109.87 pixels The number of $\mathrm{cm}$ per pixel is 0.002749

OK

Figure 3. a) Distance for calibration, b) units of the calibration, c) corresponding distance according to the selected calibration units.

Once the system has been referenced to a real spatial unit, the diameter of the Airy disk is measured by selecting the initial and final position of the diameter of the brightest disk, as shown in Figure 4a) with a dashed red. Its calculated distance in the pre-specified unit as well as in pixels will be immediately calculated (Figure $4 \mathrm{~b}$ )). The RGB level corresponding to the selected area will be also shown as in Figure 4c) demonstrating that most of the energy is concentrated in the central disk.

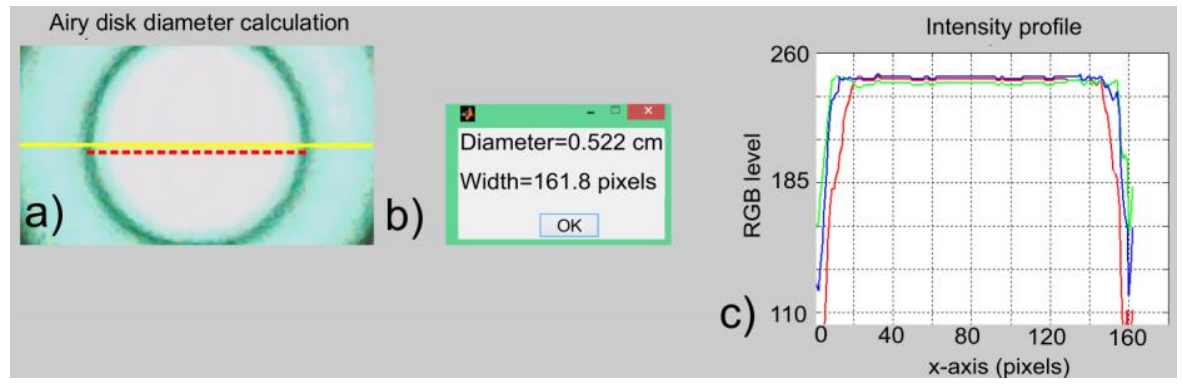

Figure 4. a) Distance for calibration, b) units of the calibration, c) corresponding distance according to the selected calibration units.

With the measurement of the disk diameter $D$, the distance $R$ and also with the nominal size of the pinhole $d$ used in the setup, it is possible to apply Equation (2) to estimate the wavelength of the illuminating laser source.

It is worth noting that Figure 4c) shows saturation in the 0-255 RGB range, for this reason the plot is cut at this maximum value. This could be avoided by reducing the optical power of the beam; however it was intentionally permitted in order to demonstrate that one of the advantages of the proposal is that the measurement of the disk diameter is independent of the saturation level as long as this does not affect the visualization of the first ring.

\section{EXPERIMENTAL RESULTS}

Figures 3-4 show a particular sample measurement, where $R=41 \mathrm{~cm}$ and $r=D / 2=0.522 / 2=0.2610 \mathrm{~cm}$, with a nominal aperture diameter $d=0.01 \mathrm{~cm}$. With the previous data the wavelength estimation corresponding to the illuminating laser source is $\lambda \approx \frac{0.01 \cdot 0.261}{1.22 \cdot 41} \approx 5.22 \times 10^{-5} \mathrm{~cm}$. 
To demonstrate the viability of the proposed method, the wavelength of a generic red and a green diode lasers with a nominal wavelength given by the manufacturer of $640 \mathrm{~nm}$ and $520 \mathrm{~nm}$ has been achieved, for a range of $R=[25,95]$ in steps of $5 \mathrm{~cm}$. The results are shown in Table 1.

Table 1. Wavelength estimations corresponding to a red and green generic laser diodes.

\begin{tabular}{|c|c|c|c|c|}
\hline $\begin{array}{c}\text { Distance } \\
(R-\mathrm{cm})\end{array}$ & $\begin{array}{c}\text { Airy } \\
\text { diameter } \\
D \text { (red } \\
\text { laser- } \\
\mathrm{cm})\end{array}$ & $\begin{array}{c}\text { Estimated } \\
\text { wavelength } \lambda \\
\text { (red laser-nm) }\end{array}$ & $\begin{array}{c}\text { Airy } \\
\text { diameter } \\
D \text { (green } \\
\text { laser- } \\
\text { cm) }\end{array}$ & $\begin{array}{c}\text { Estimated } \\
\text { wavelength } \lambda \\
\text { (green laser- } \\
\text { nm) }\end{array}$ \\
\hline 25 & 0.38 & 626.22 & 0.32 & 521.31 \\
\hline 30 & 0.45 & 625.68 & 0.38 & 520.49 \\
\hline 35 & 0.53 & 625.29 & 0.44 & 521.07 \\
\hline 40 & 0.61 & 626.02 & 0.50 & 516.39 \\
\hline 45 & 0.68 & 626.59 & 0.57 & 519.12 \\
\hline 50 & 0.75 & 622.13 & 0.63 & 518.85 \\
\hline 55 & 0.83 & 619.2 & 0.69 & 517.88 \\
\hline 60 & 0.92 & 631.14 & 0.76 & 524.59 \\
\hline 65 & 0.98 & 621.05 & 0.84 & 529.63 \\
\hline 70 & 1.07 & 626.46 & 0.88 & 519.90 \\
\hline 75 & 1.15 & 633.33 & 0.94 & 517.48 \\
\hline 80 & 1.23 & 633.19 & 1.02 & 524.59 \\
\hline 85 & 1.30 & 628.73 & 1.06 & 515.42 \\
\hline 90 & 1.39 & 633.87 & 1.14 & 521.40 \\
\hline 95 & 1.46 & 631.57 & 1.21 & 524.59 \\
\hline & & $\bar{\lambda}=627.36 \mathrm{~nm}$ & & $\bar{\lambda}_{g}=520.84 \mathrm{~nm}$ \\
\hline
\end{tabular}

In order to validate the proposal, the resulting average of the two wavelength estimations $\left(\bar{\lambda}_{r}, \bar{\lambda}_{g}\right)$ were compared with the wavelength provided by the manufacturer corresponding to the laser sources by calculating the percentage error

$$
\%_{\text {error_red }}=\left|\frac{627.36-640}{640}\right| .100=1.97 \%, \quad \%_{\text {error_green }}=\left|\frac{520.84-520}{520}\right| .100=0.16 \%
$$

\section{CONCLUSIONS}

We have presented a simple, low-cost didactic and educational method to estimate the wavelength of a laser source by using the diameter of an Airy disk, generated by the Fraunhofer diffraction phenomenon resulting from a circular aperture, without the necessity of using optical meters, devices or setups such as wavemeters, spectrometers, interferometers as well as optical fibers and diffraction gratings, to name a few, which in some cases are hard to find in optical or physics laboratories and also substantially increase the implementation expenses. In addition, the setup has been built over an ordinary non-isolated table which adds a versatility feature.

It is necessary to point out that the exposed method calculates an estimated value of the wavelength because, due to the characteristics of the environment and devices used in the setup as well as the measuring procedure that implicates a manual selection of the initial and final position of a spatial reference and of the diameter of the Airy disk (both depending on the appreciation of the user, generating the main source of error), as well as the measurement of the distance $R$, several sources of error are presented. However we strongly believe that this proposal gives a very well approximation and is perfectly suitable to be applied for didactic, educational or demonstrative purposes in topics that involve optical phenomena and concepts such as diffraction and wavelength for undergraduate or graduate students in the field of physics or wherever these concepts are related. Due to the characteristic and materials used in the proposal, this can be easily implemented in didactic laboratories or even in classrooms, which is particularly desirable when many students are presented. 
The developed windows standalone application written in MATLAB that was used in this proposal can be freely downloaded in the following link: https://drive.google.com/open?id=0Bz9Jz7_ucF7gcC1CZSOtdXRIOHc

If the MATLAB Compiler Runtime (MCR) is needed, the full package can be downloaded from: https://drive.google.com/open?id=0Bz9Jz7_ucF7gZHRQSERQWFZCMkU

\section{REFERENCES}

[1] Hobbs, P., [Building Electro-Optical Systems], John Wiley \& Sons, Inc., New York, $52-54$ (2009).

[2] Malacara, D., [Optica Básica], Fondo de Cultura Económica, México, D.F., 484-488 (2004).

[3] Hecht, E., [Optics], 4th ed. Addison Wesley, 476-481 (2001).

[4] Rivera-Ortega, U. and Dirckx, J. "On-off Laser Diode control for phase retrieval in phase-shifting interferometry," Appl. Opt. 54, 3576-3579 (2015).

[5] Fox, P. J., Scholten, R. E., Walkiewicz, M. R. and Drullinger, R. E., "A reliable, compact, and low-cost Michelson wavemeter for laser wavelength measurement," Am. J. Phys. 67, 624-630 (1999).

[6] Catunda, T., Sartori, J. and Nunes, L., "Plane wave interference: A didactic experiment to measure the wavelength of light, " Am. J. Phys. 66, 548-549 (1998).

[7] Rivera-Ortega, U., "Automated method for wavelength estimation in a two-beam interferometer based on the on-off switching of two laser-diode sources," Eur. J. Phys. 38, 1-6 (2017).

[8] White, J. D. and Scholten, R. E. "Compact diffraction grating laser wavemeter with sub-picometer accuracy and picowatt sensitivity using a webcam imaging sensor, " Rev. Sci. Instrum. 83, 1-4 (2012).

[9] Schawlow, A. L. "Measuring the wavelength of light with a Ruler," Am. J. Phys. 33, 922-923 (1965).

[10] Hecht, E [Optics], 4th ed., Addison Wesley, 467-471 (2002).

[11] Soskind, Y. G., [Field Guide to Diffractive Optics], SPIE Press, Washington, 14-16 (2011).

[12] Pedrotti, F. L. and Pedrotti, L. S., [Introduction to Optics], 2nd ed. Prentice-Hall, New Jersey, 332-334 (1993).

[13] Soskind, Y. G., [Field Guide to Diffractive Optics], SPIE Press, Washington, 28-29 (2011).

[14] Pedrotti, F. L. and Pedrotti, L. S., [Introduction to Optics], 2nd ed. Prentice-Hall, New Jersey, 335-337 (1993).

[15] Cowley, L., Laven, P. and Vollmer, M., "Rings around the sun and moon: coronae and diffraction, " Phys. Educ. 40, 51-59 (2005).

[16] James, J. F., [A Student's Guide to Fourier Transforms], Cambridge University Press, New York, 38-42 (2004).

[17]Fowles, G. R., [Introduction to Modern Optics], Dover Publications, Inc., New York, 112-125 (1975). 